\title{
A Rare Presentation of Cryptococcal Meningitis in a Treatment-Naive Patient with Sarcoidosis
}

\author{
Mehak Qureshi, Basel Abdelazeem, Ashiya Khan, Mazen Najjar \\ McLaren Health Care, Flint/Michigan State University, Flint, Michigan, USA
}

Received: 01/07/2021

Accepted: $12 / 07 / 2021$

Published: 09/08/2021

\begin{abstract}
How to cite this article: Qureshi M, Abdelazeem B, Khan A, Najjar M. A rare presentation of cryptococcal meningitis in a treatment-naive patient with sarcoidosis. EJCRIM 2021;8: doi:10.12890/2021_002755.
\end{abstract}

Conflicts of Interests: The authors declare there are no competing interests.

This article is licensed under a Commons Attribution Non-Commercial 4.0 License

\section{ABSTRACT}

Cryptococcus neoformans is an encapsulated, yeast-like fungus that commonly lives in the environment due to soil contamination by the faeces of birds, especially pigeons. Cryptococcus is an opportunistic fungal infection frequently diagnosed in immunocompromised patients with HIV, steroid use, malignancy, history of organ transplantation, or, rarely, sarcoidosis. There have been only a few reports of cryptococcus infection in sarcoidosis patients who were not on steroid treatment. Here, we highlight the importance of considering opportunistic fungal infection in asymptomatic treatment-naive sarcoidosis patients. We present a patient with a history of asymptomatic, treatmentnaive sarcoidosis who presented with headache and was diagnosed with cryptococcal meningitis in the presence of an idiopathic T-cell lymphopenia.

\section{LEARNING POINTS}

- Cryptococcus is an opportunistic fungal infection commonly diagnosed in immunocompromised patients.

- Sarcoidosis is a multi-systemic disease characterized by the formation of granulomas in various organs, most commonly found in the lungs and mediastinal lymph nodes.

\section{KEYWORDS}

Cryptococcus neoformans, meningitis, sarcoidosis

\section{INTRODUCTION}

Cryptococcus neoformans is an encapsulated, yeast-like fungus that commonly lives in the environment due to soil contamination by the faeces of birds, especially pigeons ${ }^{[1]}$. Infection with cryptococcus (either C. neoformans or $C$. gatti) is called cryptococcosis, an opportunistic fungal infection commonly diagnosed in immunocompromised patients infected with HIV ${ }^{[2]}$. The global annual incidence of cryptococcal meningitis is 220,000 , resulting in approximately 181,000 deaths every year. The low incidence of cryptococcal meningitis in immunocompetent patients is a common cause of its delayed diagnosis in that patient population ${ }^{[2]}$. The main means of protection against fungal infection is through T-cell mediated immunity. Although most cryptococcosis cases are seen in HIV-infected patients, cases have been reported in a few HIV-negative patients immunocompromised due to steroid use, malignancy, organ transplantation, or rarely, sarcoidosis ${ }^{[3]}$. We present a patient with a history of asymptomatic, treatment-naive sarcoidosis who developed cryptococcal meningitis in the presence of an idiopathic T-cell lymphopenia. 


\section{CASE DESCRIPTION}

A 46-year-old Caucasian woman presented with an 8-week history of headaches associated with low-grade fevers, chills, generalized weakness and bilateral hearing impairment. The patient's medical history was significant for biopsy-proven sarcoidosis; she was not on any chronic medication for this condition. The patient was first diagnosed with pulmonary nodules in 2014 on CT of the chest and then underwent bronchoscopy with endobronchial biopsy in 2014, which confirmed the diagnosis of sarcoidosis. However, treatment was not warranted at that time as it was a mild case. Unfortunately, the patient was subsequently lost to follow-up.

The patient was not on any immunosuppressive medications or steroids. She reported being sexually active with one partner for the last 10 years. On physical examination, her vital signs were within normal limits, and the neurological exam was significant for symmetrical hyporeflexia and decreased sensation below the knees. She did not have nuchal rigidity, and Kernig's and Brudzinski's signs were negative. Blood work, including a complete blood count, was significant for a slightly elevated WBC count of 11.64, but the comprehensive metabolic panels were within normal limits. Chest x-ray showed subtle bilateral upper lobe opacities without any consolidation (Fig. 1).

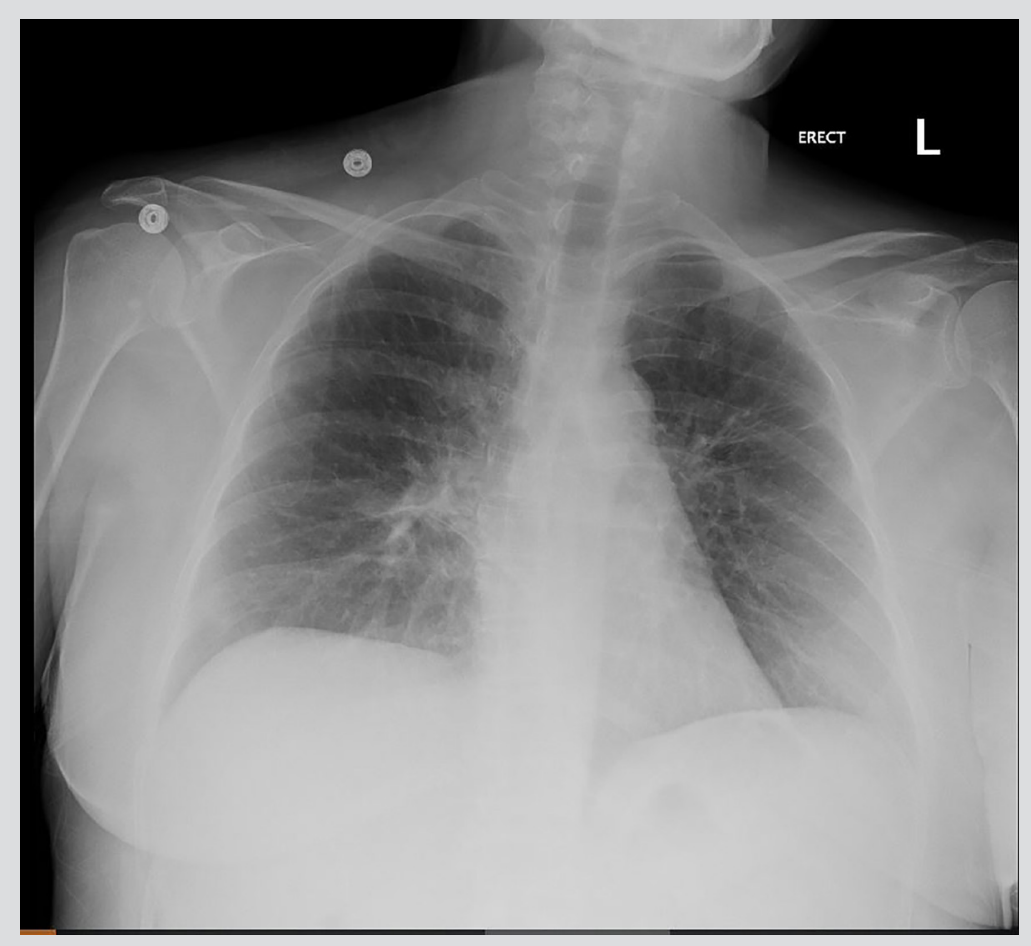

Figure 1. Chest $x$-ray showing subtle bilateral upper lobe opacities without any consolidation

The results of samples obtained from multiple lumbar punctures performed throughout hospitalization are given in Table 1. CSF samples were sent to be analysed for tuberculosis, and fungal, bacterial and viral infections. The bacterial CSF culture and AFB culture were negative, but the fungal CSF culture was positive for $\mathrm{C}$. neoformans. HIV results were negative. The erythrocyte sedimentation rate was 12 (0-20 $\mathrm{mm} / \mathrm{hr}), \mathrm{C}$-reactive protein was $0.5(0.0-0.9 \mathrm{mg} / \mathrm{dl})$ and ACE levels were elevated to 28 . Paraneoplastic evaluation for autoantibodies was performed and was negative for amphiphysin Ab, AGNA-1, ANNA-1, ANNA-2, ANNA-3, CRMP-5-IgG PCA-Tr, PCA-1 and PCA-2. Immune cell function assays were performed and revealed a normal WBC count of 5930 (4500-10,000×10\%/l) and a lymphocyte count of 900 ( $900-$ $\left.5000 \times 10^{\%} / \mathrm{I}\right)$. The CD4 count was $228(481-1793 \times 109 / \mathrm{I})$ and the CD8 count was $240\left(211-851 \times 10^{9} / \mathrm{l}\right)$ with a low CD4:CD8 ratio of 0.94 (1.00-3.90). The CD3 count was also found to be low at 435 (451-2199 $\left.\times 10^{9} / \mathrm{I}\right)$. In view of the patient's episodes of intermittent confusion, EEG was performed and was negative for any epileptogenic foci or seizures. Additionally, a repeat CT of the chest was done, which showed calcified granulomas with diffuse reticular nodular opacities and multiple lymph nodes throughout the mediastinum, with the largest in the right paratracheal region, which were stable from her previous scan in 2014.

The patient was empirically started on vancomycin, ceftriaxone and azithromycin. CSF fungal cultures were reported positive for $C$. neoformans which prompted a change to IV amphotericin B. However, the patient continued to have episodes of headaches associated with photophobia and blurry vision so she underwent serial therapeutic lumbar punctures to help relieve elevated intracranial pressures and alleviate her symptoms. She was treated with IV amphotericin B for 1 week, and once she was asymptomatic, she was discharged home on 


\begin{tabular}{|l|l|l|l|l|l|}
\hline & Day 1 & Day 3 & Day 4 & Day 6 & Day 12 \\
\hline Volume & $18 \mathrm{ml}$ & $13 \mathrm{ml}$ & $3 \mathrm{ml}$ & $6 \mathrm{ml}$ & $10 \mathrm{ml}$ \\
\hline Colour & Yellow & Clear & Clear & Clear & Clear \\
\hline Opening pressure & 5 & 400 & 10 & - & - \\
\hline Glucose & 1 & 4 & 5 & 3 & 6 \\
\hline Protein & 382 & 200 & 207 & 288 & 215 \\
\hline WBC & 9 & 2 & 26 & 7 & 21 \\
\hline Lymphocytes & 22 & - & 51 & - & 92 \\
\hline Monocytes & 20 & - & 18 & - & 3 \\
\hline Eosinophils & 6 & - & 7 & - & 7 \\
\hline $\begin{array}{l}\text { Cryptococcal } \\
\text { antigen titre }\end{array}$ & Positive; 1:1024 & Positive; 1:128 & Positive; 1:256 & Positive; 1:128 & Positive; 1:128 \\
\hline
\end{tabular}

Table 1. Analysis of cerebrospinal fluid samples obtained from the patient on hospital days 1, 3, 4, 6 and 12

hospital day 12 on fluconazole $800 \mathrm{mg}$ PO daily for at least another 8 weeks. She was also to have liver function tests and her potassium levels monitored as an outpatient while on therapy.

The patient continued to follow up with the neurology and infectious disease departments. She was seen in the infectious disease clinic 2 weeks after discharge from hospital and appeared well and had no recurrence of headache or any other symptoms. She also followed up with neurology and did not need to have any more lumbar punctures to resolve her symptoms. Pulmonology will continue to follow up with the patient in 6 months with a repeat CT scan of the chest to monitor her sarcoidosis.

\section{DISCUSSION}

Cryptococcus is an encapsulated budding yeast that can cause infection in both humans and animals worldwide. $C$. neoformans is found predominantly in the USA in soil contaminated by bird faeces, while C. gatti is most commonly found in tropical and subtropical regions ${ }^{[4]}$. Cryptococcus is transmitted through inhalation. Once it has entered the respiratory system and settled in the lung parenchyma ${ }^{[4]}$, it can then spread haematogenously to the brain, leading to cryptococcal meningitis in the infected host ${ }^{[5]}$.

T-cell mediated immunity is the most important defence mechanism against fungal infections such as cryptococcosis ${ }^{[3]}$. Sarcoidosis cryptococcal meningitis is a rare complications of sarcoidosis due to the impaired T-cell immunity resulting from the sequestration of CD4-T cells into granulomas that results from the suppression of T-cell proliferation by regulatory T-cells ${ }^{[3]}$. Sarcoidosis is associated with cellmediated immunodeficiencies such as T-cell lymphopenia, while treatment for sarcoidosis can further compromise the immune system [3]. Treatment of sarcoidosis with glucocorticoids or cytotoxic agents such as mycophenolate mofetil, a tumour necrosis factor-alpha (TNF) antagonist, causes immunosuppression and is known to increase the risk of infection ${ }^{[1]}$. Despite the CD4 T-lymphopenia and treatmentinduced immunosuppression that may result, the risk of developing opportunistic infections is not considered to be higher in patients with sarcoidosis compared with the general population ${ }^{[3,5]}$.

Diagnosing cryptococcal meningitis in a seemingly immunocompetent patient is initially difficult due to its low incidence in immunocompetent patients and its non-specific diagnostic features ${ }^{[3,5]}$. Our patient was seemingly immunocompetent with no symptoms of sarcoidosis except for a few pulmonary nodules seen on the CT scan, which were reported to be unchanged from 2014 when she was first diagnosed. 
Additionally, she had never been on any medical treatment for her sarcoidosis, nor did she report any symptoms of illness aside from the ones reported on admission. However, despite not being on any therapy, the CD4-T cell lymphopenia put her at a higher risk for contracting cryptococcal meningitis.

Before the results of her fungal culture reporting $C$. neoformans were received, she was receiving therapy for bacterial or viral meningitis, which initially delayed her treatment. The treatment of cryptococcal meningitis follows a three-step process of induction, consolidation and then maintenance (for immunosuppressed patients) ${ }^{[5]}$. Induction involves the treatment of patients with IV anti-fungal therapy, which was done with IV amphotericin B for 1 week, followed by induction and maintenance therapy with fluconazole 800 mg daily for 1 year.

In conclusion, our case illustrates the importance of considering the possibility of fungal infections in patients with a history of asymptomatic sarcoidosis despite being treatment-naive. It serves as a reminder to physicians to maintain a high level of suspicion for the disease despite unique, non-traditional presentations and patient populations. It also demonstrates a positive outcome of cryptococcal meningitis in a patient with sarcoidosis and CD4-T cell lymphopenia, which is rare in this population due to possible delays in diagnosis and a compromised immune system.

\section{REFERENCES}

1. van der Stoep JH, Sigstad E, Bredberg A. Sarcoidosis with cryptococcal infection apparently engaging only immune-privileged body compartments: a case report. BMC Infect Dis 2020;20(1):440.

2. Wagemakers A, Ang CW, Hagen F, Bot JCJ, Bomers MK, Visser MC, et al. Case report: chronic relapsing cryptococcal meningitis in a patient with low mannose-binding lectin and a low naive CD4 cell count. BMC Infect Dis 2019;19(1):846.

3. Leonhard SE, Fritz D, van de Beek D, Brouwer MC. Cryptococcal meningitis complicating sarcoidosis. Medicine (Baltimore) 2016;95(35):e4587.

4. Jain D, Najjar M,Azher Q, Bachuwa G. Cryptococcal sternal osteomyelitis in a healthy woman: a review of Cryptococcus neoformans. BMJ Case Rep 2013;2013:bcr2013009129.

5. Khattab A, Patruni S, Sealey ML. Rare presentation of cryptococcal meningitis in an immunocompetent patient. BMJ Case Rep 2019;12(5):e230003. 